\title{
A real-time XAS PEEM study of the growth of cobalt iron oxide on Ru(0001)
}

Cite as: J. Chem. Phys. 152, 074704 (2020); https://

doi.org/10.1063/1.5140886@jcp.2020.0XIDE2020.issue-1

Submitted: 02 December 2019 . Accepted: 24 January 2020 . Published Online: 18 February 2020

S. Ruiz-Gómez, A. Mandziak (D), J. E. Prieto (D), M. Aristu (D), E. M. Trapero, G. D. Soria, A. Quesada, M. Foerster (D), L. Aballe (D), and J. de la Figuera (D)

\section{COLLECTIONS}

Note: This paper is part of the JCP Special Topic on Oxide Chemistry and Catalysis.

F This paper was selected as Featured
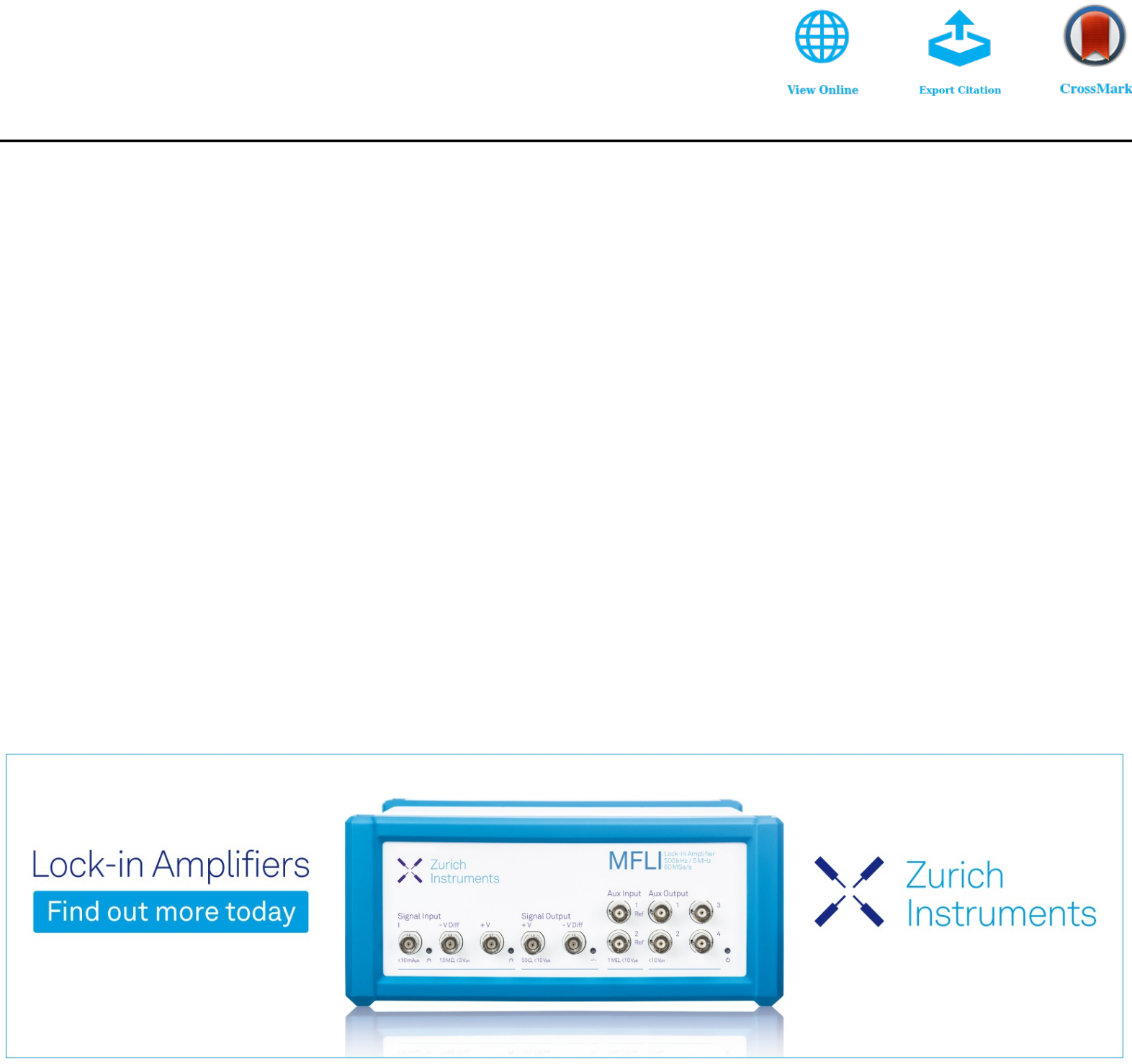


\title{
A real-time XAS PEEM study of the growth of cobalt iron oxide on $\mathrm{Ru}(0001)$
}

\author{
Cite as: J. Chem. Phys. 152, 074704 (2020); doi: 10.1063/1.5140886 \\ Submitted: 2 December 2019 - Accepted: 24 January 2020 • \\ Published Online: 18 February 2020
}

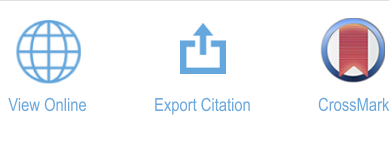

\author{
S. Ruiz-Gómez, ${ }^{1,2}$ A. Mandziak, ${ }^{3,4}$ (D) J. E. Prieto, ${ }^{3}$ (D) M. Aristu, ${ }^{3}$ (D) E. M. Trapero, ${ }^{3}$ G. D. Soria, ${ }^{3}$ A. Quesada, ${ }^{5}$ \\ M. Foerster, ${ }^{4}$ L. Aballe, ${ }^{4}$ and J. de la Figuera ${ }^{3, a)}$ (D)
}

\author{
AFFILIATIONS \\ ${ }^{1}$ Dpto. de Física de Materiales, Univ. Complutense de Madrid, Madrid E-28040, Spain \\ ${ }^{2}$ Unidad Asociada IQFR (CSIC)-UCM, Madrid E-28040, Spain \\ ${ }^{3}$ Instituto de Química Física "Rocasolano," CSIC, Madrid E-28006, Spain \\ ${ }^{4}$ ALBA Synchrotron Light Facility, CELLS, Cerdanyola del Vallés E-08290, Spain \\ ${ }^{5}$ Instituto de Cerámica y Vidrio, CSIC, Madrid E-28049, Spain
}

Note: This paper is part of the JCP Special Topic on Oxide Chemistry and Catalysis.

a) Author to whom correspondence should be addressed: juan.delafiguera@csic.es

\begin{abstract}
The growth of mixed cobalt-iron oxides on $\mathrm{Ru}(0001)$ by high-temperature oxygen-assisted molecular beam epitaxy has been monitored in real time and real space by $\mathrm{x}$-ray absorption photoemission microscopy. The initial composition is a mixed Fe-Co(II) oxide wetting layer, reflecting the ratio of the deposited materials. However, as subsequent growth of three dimensional spinel islands nucleating on this wetting layer takes place, the composition of the oxide in the wetting layer changes as iron is transferred into the spinel islands. The composition of the islands themselves also changes during growth.
\end{abstract}

Published under license by AIP Publishing. https://doi.org/10.1063/1.5140886

\section{INTRODUCTION}

Extremely high quality oxide films can be obtained by depositing metals by molecular beam epitaxy on a hot substrate, while the sample is exposed to an oxidizing agent such as molecular oxygen. This method has been used to grow many different oxide films including complex high- $\mathrm{T}_{c}$ superconductors. ${ }^{1}$ Although such a method requires, in many cases, a precise adjustment of the different dosers, for many oxides it is enough to continuously deposit the required metals on the chosen substrate. However, especially at high temperatures, the competition of kinetic and thermodynamic effects can give rise to unexpected (sometimes undesired) phases. A good example is the growth of binary and ternary oxides on metals ${ }^{2}$ with applications in magnetism or catalysis, where the metallic substrate provides an environment which is quite different from any termination of the growing oxide. A metallic substrate allows for the full range of electron-based characterization techniques to be applied to the films.
An electron-based technique that gives real-space and real-time information of the growing front of the film is low-energy electron microscopy (LEEM). ${ }^{3}$ In LEEM, the surface is imaged using the electrons reflected from the surface. The use of glancing incidence molecular beam epitaxy dosers permits depositing the material while the surface is observed with nanometer resolution. Using this technique, a surprising behavior has been discovered in metal systems, highlighting the role of alloying during growth. ${ }^{4-6}$ A LEEM instrument is also able to acquire low-energy electron diffraction (LEED) patterns and, if an x-ray source is available, to measure chemical maps by acquiring the secondary electrons emitted upon $\mathrm{x}$-ray absorption [photoemission microscopy (PEEM) ${ }^{7}$ ].

The LEEM technique has been applied to study the growth of oxides from the initial nucleation of islands to the formation of complete films. Among the oxides explored, we mention iron, ${ }^{8,9}$ cobalt, ${ }^{10}$ cerium, ${ }^{11,12}$ and praseodymium ${ }^{13}$ oxides on $\mathrm{Ru}(0001) .{ }^{14}$ Such experiments often show phase coexistence and allow for the study of the influence of growth conditions on the different phases obtained. As 
an example, consider the growth of iron oxides. ${ }^{15}$ Initially, islands nucleate with a cation(II) oxidation state $(\mathrm{FeO})$, whose thickness depends on the oxygen background pressure. ${ }^{16}$ The $\mathrm{FeO}$ islands then grow laterally until they cover the substrate completely. Then, as more material is deposited, eventually islands of spinel phase (magnetite) grow as flat micrometer-sized crystals on the top of the FeO wetting layer. ${ }^{9}$ The LEEM technique can be applied during the growth, ${ }^{8}$ complemented with structural information obtained from low-energy electron diffraction acquired with the same instrument and with chemical information determined from PEEM. The latter information is usually obtained a posteriori: after the growth is stopped, the surface is characterized structurally and chemically. For binary oxides, such a powerful combination can completely characterize the phases grown.

In ternary oxides, however, even within a single phase, the composition might not be homogeneous or more than one phase can form. In fact, especially at high temperatures, the stoichiometry can change during growth. There is then the need for acquiring chemical maps during growth. For example, mixed cobalt-iron, ${ }^{10,18}$ nickel-iron, ${ }^{19}$ and cobalt-nickel ${ }^{20}$ oxides have been studied, combining LEEM observations during growth with the LEED and PEEM information of the final surface. However, a surprising discrepancy has been observed in the first two cases. The growth mode is similar to iron oxides on $\mathrm{Ru}(0001)$ : first, islands of a mixed (II) oxide $\left(\mathrm{Co}_{x} \mathrm{Fe}_{1-x} \mathrm{O}\right.$ or $\left.\mathrm{Ni}_{x} \mathrm{Fe}_{1-x} \mathrm{O}\right)$ grow, which then coalesce to form a continuous layer. In the second stage, spinel islands $\left(\mathrm{Co}_{x} \mathrm{Fe}_{2-x} \mathrm{O}_{4}\right.$ or $\mathrm{Ni}_{x} \mathrm{Fe}_{2-x} \mathrm{O}_{4}$ ) appear. However, when the composition of the final films is measured, in all cases the ratio of the cations in the wetting layer and in the spinel islands differ from each other and from the deposited ratio. For example, for a deposited ratio of iron to the second cation of $2: 1,{ }^{18,19}$ the wetting layer presents a ratio closer to $1: 1$, while the islands are Fe rich.

To measure the evolution of the surface composition in such a multicomponent growth front, we require a method to determine the composition at the nanometer scale in real time. Here, we propose to do this by sequentially recording photoemission microscopy images ${ }^{7}$ under illumination of $\mathrm{x}$-rays with photon energies corresponding to the metal absorption edge (Co and $\mathrm{Fe}_{3}$ in the present case).

\section{EXPERIMENTAL}

The in situ $\mathrm{X}$-ray absorption experiments have been performed at the CIRCE beamline of the ALBA Synchrotron Light Facility.
The endstation has a low-energy electron microscope that can be used as a photoemission microscope. In this mode, it provides images of the energy-filtered distribution of photoelectrons with spatial and energy resolutions down to $20 \mathrm{~nm}$ and $0.2 \mathrm{eV}$, respectively. The kinetic energy of the detected photoelectrons can be selected with this energy resolution.

The substrate employed was a $\mathrm{Ru}(0001)$ single crystal. It was cleaned by cycles of annealing in oxygen at $1200 \mathrm{~K}$ in $10^{-6} \mathrm{mbar}$ of molecular oxygen followed by flashing to $1800 \mathrm{~K}$ in vacuum. The oxides were grown by depositing the proper ratio of Co and $\mathrm{Fe}$ in a background pressure of molecular oxygen with the substrate kept at a high temperature $(1300 \mathrm{~K})$. The molecular oxygen background pressure is limited by the need to have the endstation open to the synchrotron beamline when illuminating the sample with the $\mathrm{x}$-ray beam. We have used a pressure of $5 \times 10^{-7} \mathrm{mbar}$. In order to minimize the thermal drift of the manipulator, the sample was kept at a high temperature for several days. Fe and Co are deposited using dosers with an electron bombardment-heated rod of each metal surrounded by a water jacket. Their rates were initially estimated by measuring the time required to complete a pseudomorphic metallic monolayer of cobalt $(27 \mathrm{~min})$ on $\mathrm{Ru}$ and a complete bilayer of $\mathrm{FeO}$ for iron $(24 \mathrm{~min})$. This translates to an Fe:Co ratio of 1.6:1, taking into account the different lattice spacings of $\mathrm{FeO}(0.32 \mathrm{~nm})^{8,10}$ and $\mathrm{Co} / \mathrm{Ru}(0.27 \mathrm{~nm})$. The deposition rates were reduced by $50 \%$ for the particular experiments reported here.

When measuring $\mathrm{x}$-ray absorption spectroscopy (XAS) during growth, ideally one would acquire PEEM images while scanning the photon energy through the $\mathrm{L}_{3,2}$ absorption edges of Co and $\mathrm{Fe}$ repeatedly. Upon integration of a region of interest, the spatially resolved XAS spectra would be measured [see Figs. 1(b) and 1 (c) obtained after one growth sequence]. However, that is too slow to be practical as a complete energy scan of a single element can take $30 \mathrm{~min}$. Instead, only selected energies are acquired. In order to estimate changes in the composition, we need to acquire at least PEEM images at the pre-peak, peak, and post-peak regions of both $\mathrm{L}_{3}$ and $\mathrm{L}_{2}$ absorption edges of $\mathrm{Fe}$ and Co. Two different procedures were used. In the first one, only the images at several energies marked with circles in Figs. 1(b) and 1(c) were acquired. This method is fast and provides many frames, so it gives good temporal resolution. However, the reference $\mathrm{x}$-ray beam intensity is not measured, so images cannot be normalized. In the second procedure, the x-ray beam intensity is measured in synchronization with the image acquisition.
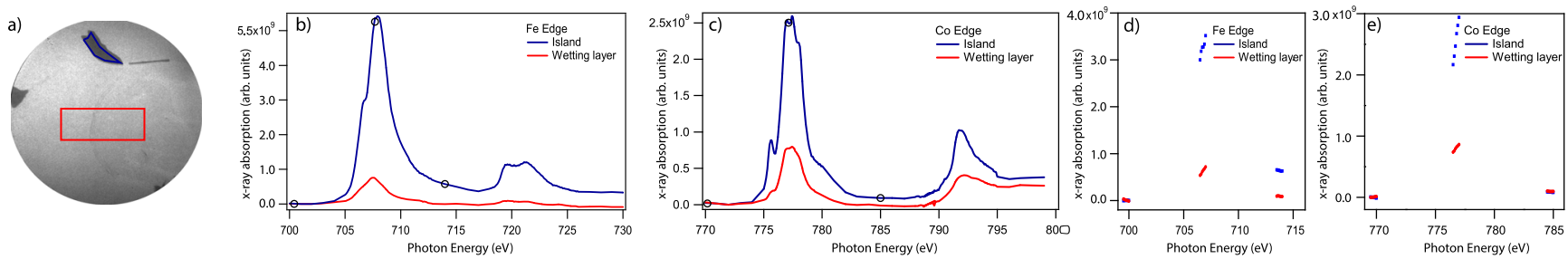

FIG. 1. (a) XAS PEEM image at $700 \mathrm{eV}$ after one growth sequence in $5 \times 10^{-7}$ mbar oxygen. The FOV of the image is $20 \mu \mathrm{m}$, the substrate temperature is $1300 \mathrm{~K}$, and the iron-cobalt ratio is 1.6:1. (b) Fe XAS spectra of the two areas indicated in (a). (c) Co XAS spectra of the same areas. The black circles show the energies used in the first sequence type of PEEM images. (d) Fe XAS spectra and (e) Co XAS spectra of the two areas indicated in (a) obtained from the second sequence type of PEEM images explained in the experimental section. 
The beam intensity is measured as a drain current in the last mirror. The synchronization at present slows down the acquisition considerably. In this mode, each energy "point" is actually a small energy range of $0.5 \mathrm{eV}$. Thus, the $\mathrm{Fe}_{3}$ prepeak position is measured with six images with photon energies in the range of $699.5-700.0 \mathrm{eV}$, the $\mathrm{Fe}_{3}$ peak with energies of $706.5-707.0 \mathrm{eV}$, and so on, as we show in Figs. 1(d) and 1(e). The energy scale of the monochromator has not been corrected to reference values, so the prepeak $(700 \mathrm{eV})$, peak $(707 \mathrm{eV})$, and post-peak $(714 \mathrm{eV})$ positions are referred instead to the spectra shown in Fig. 1.

In order to quantify the number of atoms of each cation, it is best to use the signal between the two L edges for each element. ${ }^{22}$ However, that intensity (as seen in Fig. 1) is very small and strongly affected by the background of the complete spectra. The signal-tonoise ratio is obviously much better at the $\mathrm{L}_{3}$ peaks. Thus, we select the latter in order to follow the evolution of the Fe and Co XAS signals, following the ratio of the peak and prepeak Fe and Co $\mathrm{L}_{3}$ intensities during growth. However, we note that the resonant peak intensities depend on the unoccupied d-density of states in addition to the number of $\mathrm{Co}$ and $\mathrm{Fe}$ atoms. ${ }^{22}$ As long as there are no significant changes in the unoccupied d-density of states, the $\mathrm{L}_{3}$ peak intensities should reflect the composition. This is the case in the present films, where the wetting layer corresponds to a rock-salt structure with divalent cations and the islands correspond to the spinel structure.

\section{DISCUSSION AND RESULTS}

We first proceed to grow a complete wetting layer plus some spinel islands on top, ${ }^{18}$ depositing $\mathrm{Fe}$ and $\mathrm{Co}$ at a ratio of 1.6:1. On this surface, we measure full XAS spectra of the Fe and Co L edges in order to select the photon energies to be used for the real-time growth monitoring. In Fig. 1, we show the measured Co and Fe XAS spectra of both the islands and the wetting layer. The spectra are similar to those we obtained before. However, we note that in the present case, the spectra are acquired at the growth temperature, not at room temperature.

The spectra of Fe on the island show a shoulder on the rising edge of the $\mathrm{L}_{3}$ edge and a double peak at the $\mathrm{L}_{2}$ edge. These features can be related to the spinel phases of pure iron, i.e., magnetite and maghemite. In particular, the shoulder on the $\mathrm{L}_{3}$ edge can be considered to be the intermediate of the $\mathrm{Fe}_{3} \mathrm{O}_{4}$ and the $\gamma$ $\mathrm{Fe}_{2} \mathrm{O}_{3}$ XAS spectra described by Crocombette and co-workers. ${ }^{2}$ This is expected for an iron-rich cobalt ferrite, where there is a coexistence of $\mathrm{Fe}^{3+}$ and $\mathrm{Fe}^{2+}$ in octahedral positions, as well as $\mathrm{Fe}^{3+}$ in the tetrahedral ones. We note that the spectra in Fig. 1 have been acquired with (positive) circular polarization. However, the measurement temperature is above the Curie temperature of any spinel cobalt iron oxide, ${ }^{24}$ so magnetic dichroic effects should not be observable in contrast to the case of room temperature XAS measurements. ${ }^{18}$ The Co XAS spectrum has a well defined peak on the rising edge of $\mathrm{L}_{3}$, followed by a multipeak structure and a single peak at $\mathrm{L}_{2}$. These features are characteristics of $\mathrm{Co}^{2+}$ in octahedral positions, well reproduced by XAS calculations ${ }^{25}$ and previous experimental observations. ${ }^{18}$ In particular, it is very different from $\mathrm{Co}^{2+}$ in tetrahedral positions, although the highest peak of the $\mathrm{L}_{3}$ edge might arise from some cations in such an environment. Therefore, it is clear that the island corresponds to the spinel phase of cobalt ferrite.

The wetting layer, however, shows a very different composition. For $\mathrm{Fe}$, there is a single broad peak for $\mathrm{L}_{3}$, similar to the one from $\mathrm{FeO}^{23}$ and corresponding to $\mathrm{Fe}^{2+}$ in octahedral positions. ${ }^{25}$ The Co spectrum is similar to the one of the island, corresponding to $\mathrm{Co}^{2+}$ in an octahedral environment. ${ }^{25}$ Thus, the wetting layer is composed of $\mathrm{Fe}^{2+}$ and $\mathrm{Co}^{2+}$. Experiments performed on a coverage before the wetting layer is completely closed display similar characteristics, ${ }^{10}$ which together with structural information from low-energy electron diffraction allows us to identify it as a mixed Co-Fe(II) oxide with the rock-salt structure. Selected area low-energy diffraction patterns have also been measured at room temperature in several experiments, which presented a coexistence of islands and a wetting layer. ${ }^{18}$ The results are consistent: the wetting layer shows a diffraction pattern that corresponds to the rock-salt structure, while the islands show the pattern of a spinel phase with the so-called bi-phase reconstruction. ${ }^{2}$

From the ratio of the XAS spectra between $\mathrm{L}_{3}$ and $\mathrm{L}_{2}$, we estimate that the island is iron-rich. We next image the spatial distribution of the $\mathrm{Co}$ and $\mathrm{Fe} \mathrm{L}_{3}$ peak intensities to follow the growth of the film in real time and real space. Several growth experiments have been performed at slightly different temperatures (1100 K-1300 K) obtaining similar results. In Fig. 2, we show selected frames from one of the growth sequences. In particular, we consecutively show the ratio of the $\mathrm{Fe}$ and $\mathrm{Co}_{3}$ intensities (peak-to-prepeak ratios). The growth has been followed in the past in the LEEM mode, ${ }^{18}$ but to the best of our knowledge, this is the first time that this is done in the XAS PEEM mode. Thus, in Fig. 2, brighter areas correspond to larger $\mathrm{L}_{3}$ intensities in both the Co and Fe XAS signals. In the first frame of Fig. 2, the dark gray background corresponds to the Ru substrate (covered with oxygen). Small islands are nucleated already at this stage, nearly all of them located at step edges of the $\mathrm{Ru}$ substrate. By the time the first Co image is acquired (after $13 \mathrm{~min}$ ), the islands have grown in size and some of them-the largest ones-already present a triangular shape. After $16 \mathrm{~min}$, a large island with a width of $0.7 \mu \mathrm{m}$ is visible. The now clearly triangular islands mostly have the same orientation when grown on the same terrace but can have opposite orientations in different terraces. This effect likely reflects the different termination of the hcp $\mathrm{Ru}$ terraces when separated by an odd number of atomic steps. At the frame corresponding to $19 \mathrm{~min}$, there is a clear difference in contrast at some of the edges of the growing islands near the topleft area (marked with yellow arrows): the edges are white, while the islands are light gray. This, however, does not reflect a change in the Co composition of the growing islands. Rather, it is a consequence of the slow acquisition time: each image shown in the figure is the average of ten frames, each corresponding to an exposure of $1 \mathrm{~s}$. During acquisition, the film keeps growing. Inspecting the individual frames, the different edge contrast is observed to arise from areas of the film where the islands are growing between the time to acquire the prepeak and the peak intensity images. It is, thus, obvious that not all regions grow at the same rate. Instead, the growth rate is quite uneven, with only specific areas growing so fast that they are easily observable by the change in contrast at the island edge, either in the $\mathrm{Fe}$ or the $\mathrm{Co}_{3}$ edge images. Those fast growing regions are usually the sides of triangular regions. Eventually, the islands coalesce, leaving only some triangular holes in between, 
Fe L3
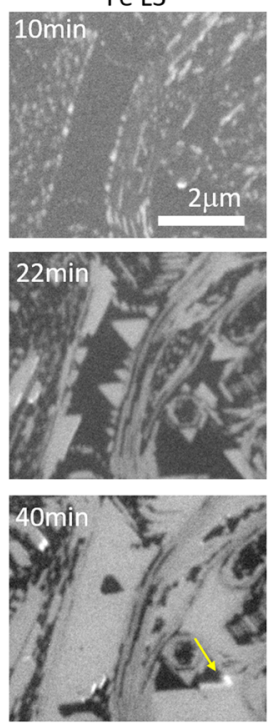

Co L3
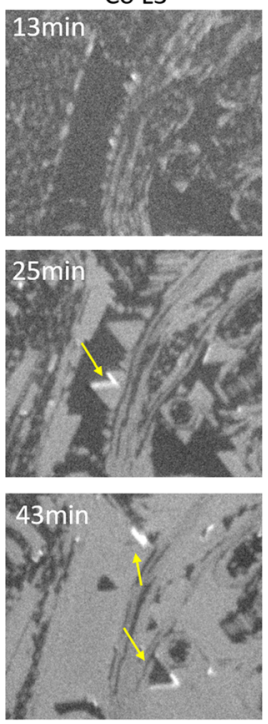

Fe L3
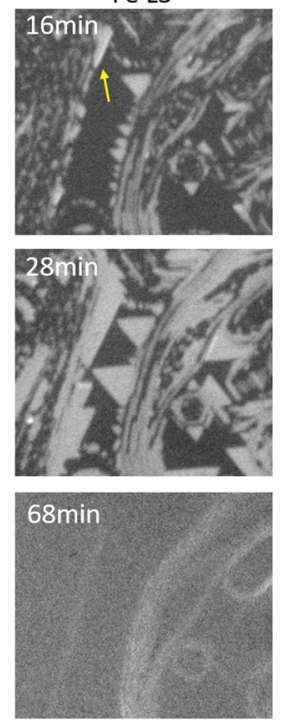

Co L3

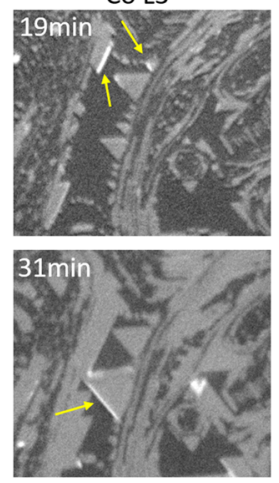

$70 \mathrm{~min}$
FIG. 2. XAS PEEM images at the ratio of the $F e L_{3}$ and $C_{0} L_{3}$ absorption edge peak to the respective pre-peak intensities at different stages of the ironcobalt growth. The time elapsed since the beginning of the growth is indicated in the upper left label on each image. Some areas of fast growth (as explained in the main text) are marked with arrows. which finally close giving rise to a complete film. Apart from some differences in contrast in stepped areas, the complete layer seems to be uniform in composition. The oxide that completely covers the surface at the last panel of Fig. 2 corresponds to the so-called wetting layer, the $\mathrm{Fe}(\mathrm{II}), \mathrm{Co}(\mathrm{II})$ oxide with the rock-salt structure. It is two layers high from previous experiments performed under similar conditions. $^{10,18}$

We next present in Fig. 3 the ratio of the Co and Fe peaks as a function of time as determined from a sequence of images in which the reference $\mathrm{x}$-ray beam flux was measured. The ratio of the $\mathrm{L}_{3}$ intensities at the Co and Fe edges is presented integrated at four different areas of the surface: one corresponding to the spinel islands that nucleate after the completion of the wetting layer and three areas corresponding to the wetting layer at different distances of the islands. Note that between frames 7 and 8 , one of the spinel islands disappears. This might be due to an Oswald ripening effect in which smaller islands are unstable towards the growth of larger ones. In previous work, ${ }^{18}$ we have presented spinel islands with a regular triangular shape. However, in those studies, the sample was observed after cooling down to room temperature and it could be explored at will in order to locate large islands. The nucleation rate at the high temperatures employed is so low that often
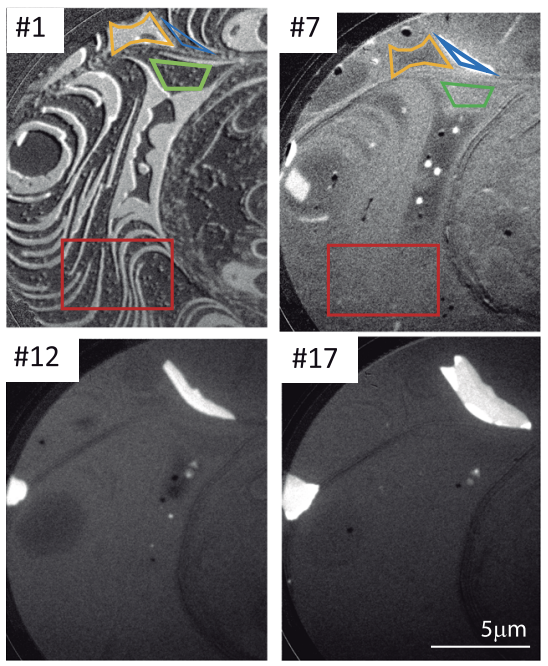

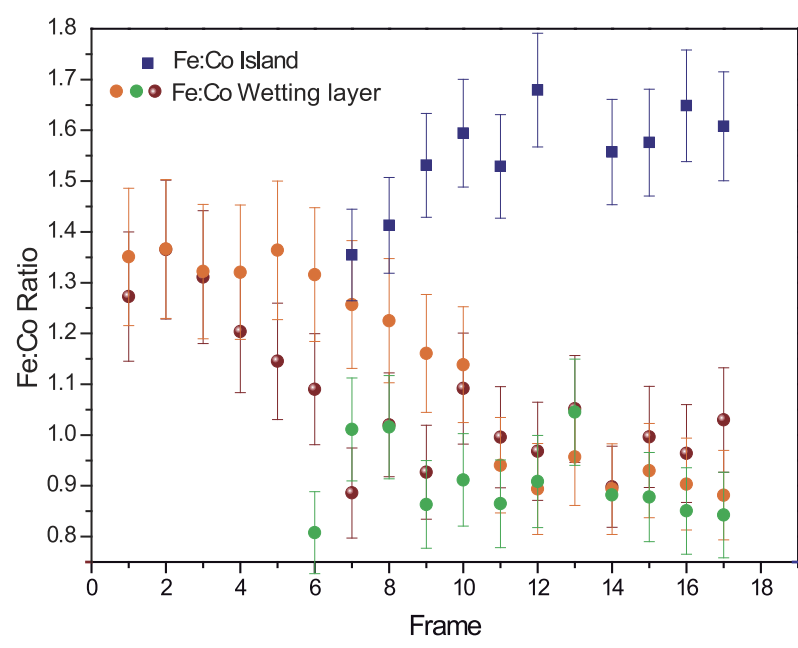

FIG. 3. Right panel: estimated composition of different regions of the wetting layer (marked in the images on the left) and the spinel islands during growth at $1300 \mathrm{~K}$. The ratio of the peak-to-prepeak intensity at the $\mathrm{Co}$ and $\mathrm{Fe}_{3}$ edges is shown at the wetting layer and at the spinel island (nucleated after frame 6). Left panel: frames extracted from the movie recorded during the growth of cobalt iron oxides on Ru(0001). They correspond to PEEM images with the photon energy at the Co $L_{3}$ absorption edge. 
there is no nucleation event in a field of view (FOV) of tens of micrometers. In our case, we intentionally imaged the same area to have a record of the evolution through the nucleation of spinel islands. The spinel islands within the field of view have a more irregular shape, which might be related to the local step density at their location.

When the wetting layer starts to grow (Fig. 3, frame 1), the $\mathrm{Fe} / \mathrm{Co} \mathrm{L}_{3}$ ratio stays close to a value of 1.3 independently of the region. This ratio should, thus, correspond to the deposited material. The deviation from the value due to the pre-calibration of the dosers (1.6:1) can be understood from the fact that the peak maxima were used instead of the absorption jump, as discussed in the experimental section. The error bars are reasonable considering that the values are extracted from quick spectra from micrometer-sized regions 2 atomic layers thick. ${ }^{18}$ Once the wetting layer is nearly complete (Fig. 3, frame 7), regions with different composition are visible within the wetting layer. The regions of the surface that were covered sooner are rich in $\mathrm{Fe}$ (region marked with an orange line), while the last regions to fill in (green rectangle) grow with the final composition of the wetting layer (less rich in Fe). This agrees with previous observations of segregation into Co-rich and Co-poor regions in the rock-salt oxide (Ref. 10). About the same time that the wetting layer is completed, the spinel islands nucleate (same blue outline in Fig. 3, frame 7). After nucleation of the spinel islands, the composition of the wetting layer continues to evolve: previously Fe-rich areas loose $\mathrm{Fe}$ until the $\mathrm{Fe} / \mathrm{Co} \mathrm{L}_{3}$ ratio is close to 1 over the whole layer, i.e., less Fe content than the deposited ratio. The extra Fe content is captured by the spinel islands, which become more iron-rich in turn. The composition of the spinel island also evolves towards a constant value upon further deposition.

In LEEM sequences (not shown ${ }^{8}$ ), it has been observed that sudden changes in contrast in the wetting layer are detected before the nucleation of spinel islands. Given the lack of such sudden changes in the present XAS movies, those changes have to be ascribed to either stacking or reconstruction changes, but not to modifications of the composition.

For Fe-only oxides, the phase diagram does not predict the existence of an oxide with the rock-salt structure ${ }^{28}$ but instead indicates that the most stable oxide at our growth conditions should be the iron spinel, i.e., magnetite. Clearly, as argued by Ketteler and Ranke, ${ }^{29}$ there is initially a kinetic barrier for the nucleation of the spinel phase, but once it nucleates, it is more stable than a multilayer FeO film. So, it is reasonable that iron moves toward the spinel islands once the latter phase nucleates. Why this happens only for $\mathrm{Fe}$ and not for Co can be understood from the bulk phase diagram of the complete $\mathrm{Co}-\mathrm{Fe}-\mathrm{O}$ system, ${ }^{30}$ which shows that at the growth temperature for a Co-rich oxide, the rock-salt phase is the stable one. We look forward to future work on detailed simulations considering the chemical potential of the different phases.

\section{CONCLUSIONS}

We have presented sequences of photoemission microscopy images, revealing the evolution of the stoichiometry during the growth of a thin film of mixed Fe-Co oxides. The experiments prove that the technique is capable of monitoring changes in the composition of complex oxides during the nucleation and growth of different phases on the surface. Confirming previous results from post-growth analysis, we could follow the wetting layer composition changes when spinel islands nucleate and start to grow, becoming Co-rich, relative to the deposited Fe/Co ratio. Furthermore, the composition of the spinel islands themselves is also found to evolve with time, becoming rich in Fe. These observations highlight the complexity of oxide growth at high temperatures where different phases compete and indicate the need for spatially and time resolved composition maps in order to fully understand the process.

\section{ACKNOWLEDGMENTS}

This work was supported by the Spanish Ministry of Science, Innovation and Universities through Project Nos. RTI2018095303-B-C51, RTI2018-095303-B-C52, and RTI2018-095303-BC53 (MCIU/AIE/FEDER, EU), by the European Commission through H2020 Project No. 720853 (Amphibian), by the CSIC PIE Project No. 190344, and by the Regional Government of Madrid through Project No. P2018/NMT-4321 (NANOMAGCOST). These experiments were performed at the CIRCE beamline of the ALBA Synchrotron Light Facility. A.M. acknowledges support from the CSIC-ALBA agreement.

\section{REFERENCES}

${ }^{1}$ D. G. Schlom, L. Chen, X. Pan, A. Schmehl, and M. A. Zurbuchen, J. Am. Ceram. Soc. 91, 2429 (2008).

${ }^{2}$ H.-J. Freund and G. Pacchioni, Chem. Soc. Rev. 37, 2224 (2008).

${ }^{3}$ E. Bauer, Surface Microscopy with Low Energy Electrons (Springer Berlin Heidelberg, 2014).

${ }^{4}$ A. K. Schmid, N. C. Bartelt, and R. Q. Hwang, Science 290, 1561 (2000).

${ }^{5}$ J. B. Hannon, J. Sun, K. Pohl, and G. L. Kellogg, Phys. Rev. Lett. 96, 246103 (2006).

${ }^{6}$ N. Rougemaille, F. El Gabaly, R. Stumpf, A. Schmid, K. Thürmer, N. Bartelt, and J. de la Figuera, Phys. Rev. Lett. 99, 106101 (2007).

${ }^{7}$ C. M. Schneider and G. Schönhense, Rep. Prog. Phys. 65, 1785 (2002).

${ }^{8}$ B. Santos, E. Loginova, A. Mascaraque, A. K. Schmid, K. F. McCarty, and J. de la Figuera, J. Phys.: Condens. Matter 21, 314011 (2009).

${ }^{9}$ M. Monti, B. Santos, A. Mascaraque, O. R. de la Fuente, M. A. Niño, T. O. Menteş, A. Locatelli, K. F. McCarty, J. F. Marco, and J. de la Figuera, Phys. Rev. B 85, 020404 (2012).

${ }^{10}$ L. Martín-García, A. Quesada, L. Pérez, M. Foerster, L. Aballe, and J. de la Figuera, Phys. Procedia 85, 12 (2016)

${ }^{11}$ J. I. Flege, J. Höcker, B. Kaemena, T. O. Menteş, A. Sala, A. Locatelli, S. Gangopadhyay, J. T. Sadowski, S. D. Senanayake, and J. Falta, Nanoscale 8, 10849 (2016).

${ }^{12}$ J. I. Flege, B. Kaemena, S. D. Senanayake, J. Höcker, J. T. Sadowski, and J. Falta, Ultramicroscopy 130, 87 (2013)

${ }^{13}$ J. Höcker, J.-O. Krisponeit, J. Cambeis, A. Zakharov, Y. Niu, G. Wei, L. C. Ciacchi, J. Falta, A. Schaefer, and J. I. Flege, Phys. Chem. Chem. Phys. 19, 3480 (2017).

${ }^{14}$ J. I. Flege and D. C. Grinter, Prog. Surf. Sci. 93, 21 (2018).

${ }^{15}$ G. S. Parkinson, Surf. Sci. Rep. 71, 272 (2016).

${ }^{16}$ I. Palacio, M. Monti, J. F. Marco, K. F. McCarty, and J. de la Figuera, J. Phys.: Condens. Matter 25, 484001 (2013).

${ }^{17}$ M. Monti, B. Santos, A. Mascaraque, O. R. de la Fuente, M. A. Niño, T. O. Menteş, A. Locatelli, K. F. McCarty, J. F. Marco, and J. de la Figuera, J. Phys. Chem. C 116, 11539 (2012).

${ }^{18}$ L. Martín-García, A. Quesada, C. Munuera, J. F. Fernández, M. García-Hernández, M. Foerster, L. Aballe, and J. de la Figuera, Adv. Mater. 27, 5955 (2015). 
${ }^{19}$ A. Mandziak, J. de la Figuera, S. Ruiz-Gómez, G. D. Soria, L. Pérez, P. Prieto, A. Quesada, M. Foerster, and L. Aballe, Sci. Rep. 8, 17980 (2018).

${ }^{20}$ A. Mandziak, G. D. Soria, J. E. Prieto, P. Prieto, C. Granados-Miralles, A. Quesada, M. Foerster, L. Aballe, and J. de la Figuera, Sci. Rep. 9, 13584 (2019).

${ }^{21}$ L. Aballe, M. Foerster, E. Pellegrin, J. Nicolas, and S. Ferrer, J. Synchrotron Radiat. 22, 745 (2015).

${ }^{22}$ J. Stöhr and H. C. Siegmann, Magnetism: From Fundamentals to Nanoscale Dynamics, Illustrated ed. (Springer, 2006).

${ }^{23}$ J. P. Crocombette, M. Pollak, F. Jollet, N. Thromat, and M. Gautier-Soyer, Phys. Rev. B 52, 3143 (1995).
${ }^{24}$ V. A. M. Brabers, Handbook of magnetic materials (1995), Vol. 8, p. 189.

${ }^{25}$ J. A. Moyer, C. A. F. Vaz, E. Negusse, D. A. Arena, and V. E. Henrich, Phys. Rev. B 83, 035121 (2011).

${ }^{26}$ N. Spiridis, K. Freindl, J. Wojas, N. Kwiatek, E. Madej, D. Wilgocka-Šlęzak, P. Dróżdż, T. Šlęzak, and J. Korecki, J. Phys. Chem. C 123, 4204 (2019).

${ }^{27}$ J. de la Figuera, J. Puerta, J. Cerda, F. E. Gabaly, and K. McCarty, Surf. Sci. 600, L105 (2006).

${ }^{28}$ G. Ketteler, W. Weiss, W. Ranke, and R. Schlogl, Phys. Chem. Chem. Phys. 3, 1114 (2001).

${ }^{29}$ G. Ketteler and W. Ranke, J. Phys. Chem. B 107, 4320 (2003).

${ }^{30}$ W.-W. Zhang and M. Chen, Calphad 41, 76 (2013). 